\title{
Treatment of Graves' ophthalmopathy with an in-house Phosphorus-32 source: Initial clinical observations
}

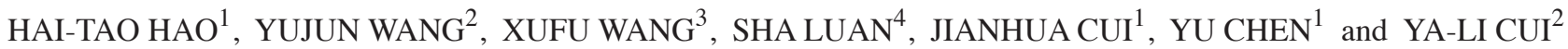 \\ ${ }^{1}$ Department of Nuclear Medicine, The Red Cross Hospital of Heilongjiang, Harbin, Heilongjiang 150040; \\ ${ }^{2}$ Department of Nuclear Medicine, Cancer Hospital of Harbin Medical University, Harbin, Heilongjiang 150081; \\ ${ }^{3}$ Department of Nuclear Medicine, The Affiliated Hospital of Qingdao University, Qingdao, Shandong 266003; ${ }^{4}$ Department \\ of Nuclear Medicine, The 4th Affiliated Hospital of Harbin Medical University, Harbin, Heilongjiang 150001, P.R. China
}

Received May 4, 2016; Accepted March 17, 2017

DOI: $10.3892 /$ etm.2017.4911

\begin{abstract}
The objective of the present study was to observe the therapeutic effect of radiation delivered via a ${ }^{32} \mathrm{P}$ source on Graves' ophthalmopathy. $\mathrm{A}^{32} \mathrm{P}$ solution was injected into a 10 -ml vacuum flask held inside a lead container. A window was cut in the lead, generating a treatment beam. Radiation was given to four areas: The upper and lower orbit (covering $\sim 1 / 3$ of the eyelid) and the inner and outer canthus. Each site received 10 daily doses of $20 \mathrm{cGy}$. Proptosis was measured by an exophthalmometer and the palpebral aperture was determined with a ruler. Measurements were taken before and after the treatment. After 5 days of treatment, the patient displayed a significant improvement, and by 10 days, the average reduction of proptosis in Graves' ophthalmopathy was $3.36 \pm 1.73 \mathrm{~mm}$ for the left and $3.05 \pm 2.04 \mathrm{~mm}$ for the right eyes. The treatment was effective in all patients, who uniformly reported rapid pain relief. Conjunctival congestion and eyelid edema also improved significantly. However, only $50 \%$ of patients showed improved diplopia after treatment, which was poor compared with other symptoms. No obvious side effects were found in the subsequent follow-up. In conclusion, ${ }^{32} \mathrm{P}$ brachytherapy for Graves' ophthalmopathy was simple and effective, with few side effects, and should be considered as a promising therapy.
\end{abstract}

Correspondence to: Professor Ya-Li Cui, Department of Nuclear Medicine, Cancer Hospital of Harbin Medical University, 150 Haping Street, Harbin, Heilongjiang 150081, P.R. China E-mail: 13936500552@126.com

Dr Xufu Wang, Department of Nuclear Medicine, The Affiliated Hospital of Qingdao University, 16 Jiangsu Road, Qingdao, Shandong 266003, P.R. China

E-mail: wangxufu@sina.com

Key words: ophthalmopathy, Graves' disease, radiation, Phosphorus-32

\section{Introduction}

Ophthalmopathy is a common complication of Graves' hyperthyroidism, affecting 50-60\% of patients (1). While most patients recover or at least show significant improvement without special treatment, certain individuals do not and may in fact experience continued deterioration after successful anti-hyperthyroidism therapy.

The diagnosis of Graves' ophthalmopathy is straightforward, based upon history and physical examination (2). The common symptoms of Graves' ophthalmopathy are eye pain, photophobia, tears, dry eyes, foreign body sensation, vision loss, severe exophthalmos (which can cause eye closure difficulty), conjunctival congestion, conjunctival ulceration and even blindness. If the extraocular muscle is involved, it can lead to a squint (2).

To control ophthalmopathy, glucocorticoids are commonly given, but this therapy has the significant side effect of immunosuppression. Retrobulbar radiotherapy has been shown to be a satisfactory treatment (3-5), particularly suitable for patients who cannot tolerate or respond to glucocorticoids. Rajendram et al (6) reviewed nine potential studies and included five trials with a total of 244 participants. It was indicated that orbital radiotherapy was more effective than sham radiotherapy for the treatment of mild-to-moderate Graves' ophthalmopathy. In a single trial, no difference between radiotherapy and steroid monotherapy was found (7). Tsujino et al (8) studied 121 cases of patients with Graves' ophthalmopathy receiving radiotherapy, and the overall clinical response was evaluated as excellent in 17 patients (14\%), good in $65(54 \%)$, fair in $31(25 \%)$, no response in $7(6 \%)$, and worse in $1(1 \%)$. No long-term complications related to radiotherapy were observed, while a limited response was noted for proptosis. Retrobulbar irradiation has been reported to be a safe and effective treatment for progressive Graves' ophthalmopathy (9). However, the expense associated with a radiotherapy units represents a major barrier to this treatment, and uninsured poor populations in developing countries may not benefit from this therapeutic strategy.

In the present study, a ${ }^{32} \mathrm{P}$ sealed source was used to deliver therapeutic radiation to the eye and its therapeutic efficacy was 
tested. ${ }^{32} \mathrm{P}$ radiation differs from an accelerator-generated electron beam in that the energy of the $\beta$-particles is reduced. If ${ }^{32} \mathrm{P}$ can be successfully deployed, the treatment process will become simple and feasible. The present study aimed to determine the clinical effects of brachytherapy with ${ }^{32} \mathrm{P}$ on ophthalmopathy.

\section{Patients and methods}

Patients. This study was preapproved by the hospital institutional review boards of The Red Cross Hospital of Heilongjiang Province and Harbin Medical University (Harbin, China). To exclude the influence of hyperthyroidism on ophthalmopathy, individuals with unresolved hyperthyroidism were excluded from the study; resolved disease was defined as stable $T_{3}$ and $T_{4}$ levels and within the normal range for at least 6 months, or oral intake of Euthyrox. The degress of ophthalmopathy was greater than moderate in all patients enrolled.

From January 2009 to January 2013, a total of 30 patients (13 males and 17 females) who had previously been treated for hyperthyroidism were referred and enrolled in the study and treated for ophthalmopathy at The Red Cross Hospital of Heilongjiang Province (Harbin, China). The average age was 37.7 years (range, 27-55). Of the 30 patients, 16 were treated with ${ }^{131} \mathrm{I}, 6$ by surgery and 7 by oral methimazole. Of the 16 patients receiving ${ }^{131} \mathrm{I}, 4$ displayed ophthalmopathy before their hyperthyroidism was controlled. In the other 12 cases, ophthalmopathy developed over a period of 6-48 months after their hyperthyroidism was resolved. Patients treated with methimazole displayed ophthalmopathy 6-24 months after hyperthyroidism was controlled, while it arose in those receiving surgery at 12-36 months.

Device and treatment protocol. $\mathrm{A}^{32} \mathrm{P}$ irradiation source was generated by injecting ${ }^{32} \mathrm{P}$ solution into a $10-\mathrm{ml}$ vacuum flask placed in a lead container with a window to generate a treatment beam (Fig. 1A).

Four points were selected in each eye for radiation: The upper and lower eye rims (not exceeding 1/3 of the eyelid) and the interior and exterior canthi. During treatment, the treatment cover was used, so that only the designated area was exposed to radiation (Fig. 1B). The radiation dose (D) was calculated according to the formula $\mathrm{D}=1,770 \times \mathrm{A} / \mathrm{S} \times 0.93$, where $\mathrm{A}$ is the activity of the source and $\mathrm{S}$ is the effective treatment area (10). In the present study, $10 \mathrm{mCi}$ of ${ }^{32} \mathrm{P}$ (HTA Co., Ltd., Beijing, China) in $1 \mathrm{ml}$ saline was sealed in the vial and each site received a total treatment dose of 200cGy delivered as daily 20cGy fractions over 10 days.

Two independent measurements of exophthalmos were made daily in all patients prior to therapy. As a control, the exophthalmos was measured in 30 thyroid disease-free volunteers recruited during annual physical examinations at The Red Cross Hospital of Heilongjiang Province (male-to-female ratio, 1:1; mean age, 37.5 years). The palpebral fissure was measured manually with an opaque hard plastic ruler.

All patients were followed up on an outpatient basis from 3-12 months after treatment.

Statistical analysis. SPSS 17.0 software (SPSS, Inc., Chicago, IL, USA) was used for statistical analyses. Comparisons between healthy volunteers and patients were made by Student's t-test.
Table I. Comparison of right end left eye protrusion (mm) in the patient group $(n=30)$ with normal volunteers $(n=30)$ prior to treatment.

\begin{tabular}{lcc}
\hline Side & Patients & Volunteers \\
\hline Right & $18.17 \pm 4.60$ & $11.89 \pm 2.51$ \\
Left & $17.68 \pm 5.16$ & $11.89 \pm 2.51$ \\
\hline
\end{tabular}

Values are expressed as the mean \pm standard deviation. For the right and left eyes, differences between the patient group and volunteer group were statistically significant $(\mathrm{P}<0.05)$.

Table II. Comparison of exophthalmos ( $\mathrm{mm}$ ) in the right $(n=30)$ and left eye $(n=30)$ prior to and at 5 and 10 days after the start of the treatment.

\begin{tabular}{lccc}
\hline Side & Baseline & 5 days & 10 days \\
\hline Right & $18.17 \pm 4.60$ & $15.74 \pm 5.01$ & $14.64 \pm 4.80$ \\
Left & $17.68 \pm 5.16$ & $15.28 \pm 5.18$ & $14.32 \pm 4.87$ \\
\hline
\end{tabular}

Values are expressed as the mean \pm standard deviation. For the left and right eyes, each time-point represents a statistically significant improvement compared to the preceding one $(\mathrm{P}<0.05)$.

Table III. Effect of treatment on the palpebral fissure in the patient cohort $(n=30)$.

Time-point

Palpebral fissure width (mm)

\begin{tabular}{ll}
\hline Before treatment & $10.54 \pm 2.18$ \\
After treatment & $10.16 \pm 1.53$ \\
\hline
\end{tabular}

Values are expressed as the mean \pm standard deviation. Treatment induced a statistically significant reduction in the palpebral fissure width $(\mathrm{P}<0.05)$.

Comparisons before and after treatment and between the 5th and 10th treatment were analyzed with the paired $\mathrm{t}$-test. $\mathrm{P}<0.05$ was considered to indicate a statistically significant difference.

\section{Results}

${ }^{32} P$ therapy reduces exophthalmos in patients with Graves' ophthalmopathy. The degree of exophthalmos of patients prior to therapy was significantly higher than that in healthy volunteers (Table I). Five days after the start of ${ }^{32} \mathrm{P}$ irradiation, a reduction in exophthalmos was observed in 28 out of 30 patients, with an overall reduction of $3.36 \pm 1.73 \mathrm{~mm}$ for the left and $3.05 \pm 2.04 \mathrm{~mm}$ for the right eye. A significant reduction in exophthalmos after 5 and 10 days of treatment was found, as shown in Table II.

${ }^{32} P$ therapy reduces Graves' ophthalmopathy symptoms and palpebral fissure width. The fissure widths prior to and after therapy are shown in Table III. One day after treatment, most 
A
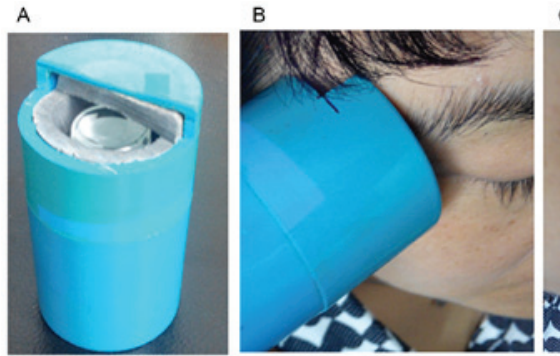

Figure 1. (A) The in-house ${ }^{32} \mathrm{P}$ source; (B and C) Examples of the therapeutic procedure.

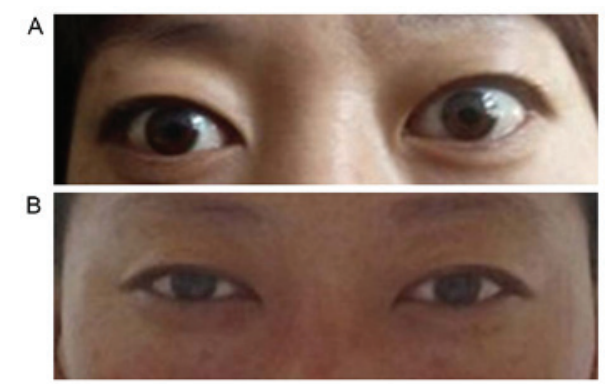

Figure 2. Graves' ophthalmopathy following external therapy with an in-house ${ }^{32} \mathrm{P}$ source in a 33-year old female. (A) Prior to therapy; (B) 12 months after treatment.

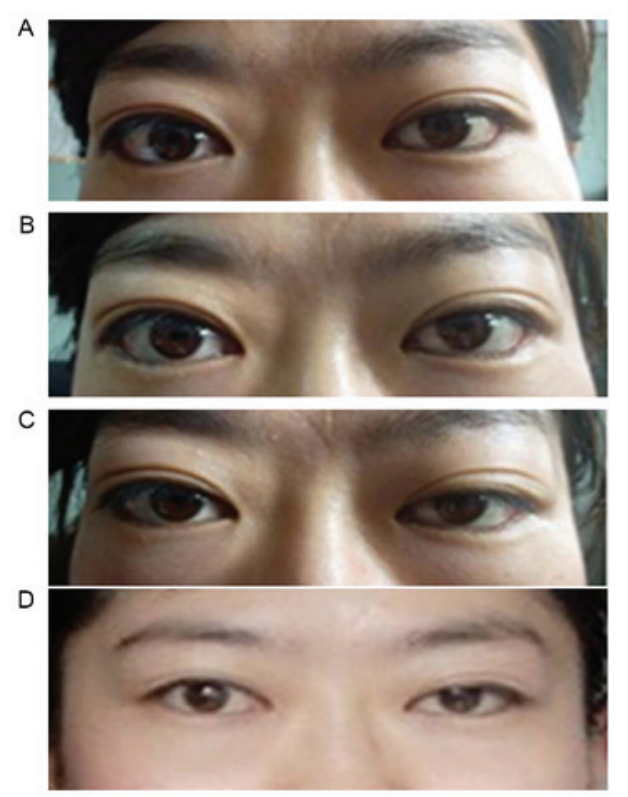

Figure 3. Graves' ophthalmopathy following external therapy with an in-house ${ }^{32} \mathrm{P}$ source in a 27-year old female. (A) Prior to therapy; (B) $24 \mathrm{~h}$ after initial therapy; (C) 10 days after the start of therapy (after receiving final dose 8 Gy for one eye); (D) 3 months after therapy.

patients had a narrow palpebral fissure. Of the 30 patients, 21 reported serious pain and 26 displayed eye swelling prior to ${ }^{32} \mathrm{P}$ therapy. Eyeball pain and swelling were generally improved after the first treatment. In addition, photophobia and tearing were generally improved on the day after the treatment started in 27 patients. Sixteen patients with gritting showed an improvement by 5-7 days after initial radiation. The response rates of these symptoms to treatment are summarized in Table IV.

Case presentations. Fig. 2 shows a 33-year-old female with swelling, pain, photophobia, tearing, exophthalmos, widened palpebral fissure and ocular proposes prior to therapy. The degree of exophthalmos was $14 \mathrm{~mm}$ for the left and $12 \mathrm{~mm}$ for the right eye. After treatment, symptoms such as swelling, pain, photophobia and tearing disappeared and the exophthalmos was reduced to $10 \mathrm{~mm}$ in each eye. The patient remained asymptomatic after 14 months of follow-up.

Fig. 3 illustrates the effect of therapy on a 27 -year-old female. Prior to therapy, when the patient presented with photophobia and tearing, the degree of exophthalmos in the left and right eyes was 17 and $18 \mathrm{~mm}$, respectively. Following ${ }^{32} \mathrm{P}$ therapy, swelling, photophobia and tearing disappeared, the degree of exophthalmos decreased to $13 \mathrm{~mm}$ and the patient remained symptom-free over 1 year of follow-up.

Fig. 4 shows the follow-up images of a 53-year-old male who suffered from severe pain and required weekly mannitol dehydration therapy. The patient had the sensation of foreign bodies in each eye, was unable to close his eyes and consequently needed to wear an eyeshade at night. He also experienced conjunctival congestion, photophobia and tearing prior to ${ }^{32} \mathrm{P}$ therapy. The degree of exophthalmos in his right and left eyes was 21 and $25 \mathrm{~mm}$, respectively. After one fraction of therapy, all the symptoms listed above disappeared and the conjunctival congestion was also obviously improved. He was able to close his eyes and no longer required an eye shade, and the degree of exophthalmos in the right and left eye was decreased to 19 and $20 \mathrm{~mm}$, respectively. A stable state was observed in a follow-up visit 4 months later.

Fig. 5 shows a 44-year-old male with photophobia, tearing, swelling and pain. His vision in each eye was decreased and eyes were extruded; he was not able to close his upper and lower right eyelids while sleeping. Prior to treatment, the degree of exophthalmos in the left and right eye was 20 and $15 \mathrm{~mm}$, respectively. The palpebral fissure of the left and right eyes was 10 and $14 \mathrm{~mm}$, respectively. After treatment, photophobia, tearing and pain disappeared, and the vision in each eye improved. The gap between the upper and lower eyelids of the right eye when closed for sleeping was clearly narrowed. The degree of exophthalmos in the left and right eye was 16 and $13 \mathrm{~mm}$, respectively. The palpebral fissure of the left and right eyes was 7 and $9 \mathrm{~mm}$, respectively. Four months later, the photophobia and tearing reappeared but was obviously reduced compared to that prior to treatment. Pain was absent and the degree of exophthalmos in the left and right eye was 17 and $13 \mathrm{~mm}$, respectively. Four months after treatment, the palpebral fissure width was unchanged from that at the end of treatment.

Side effects. The side effects of ${ }^{32} \mathrm{P}$ irradiation were minimal, and none of the 30 patients displayed skin hyperpigmentation in the irradiated sites. One patient experienced an increased level of pain, which disappeared 3 days later, and one patient suffered from a transient increase in diplopia. At follow-up, ophthalmopathy had deteriorated in only one out of 30 patients which was due to a recurrence of hyperthyroidism (Fig. 5). 
Table IV. Symptom improvement at 1 month after treatment.

\begin{tabular}{|c|c|c|c|c|c|c|}
\hline \multirow[b]{2}{*}{ Symptom } & \multirow[b]{2}{*}{$\mathrm{N}$} & \multirow[b]{2}{*}{ Disappearance } & \multicolumn{3}{|c|}{ Improvement } & \multirow[b]{2}{*}{ Efficiency (\%) } \\
\hline & & & Significant & Partial & None & \\
\hline Pain & 21 & 20 & 1 & 0 & 0 & 100 \\
\hline Eye swelling & 26 & 25 & 1 & 0 & 0 & 100 \\
\hline Diplopia and strabismus & 12 & 6 & 0 & 0 & 6 & 50 \\
\hline Photophobia and tearing & 27 & 10 & 13 & 4 & 0 & 100 \\
\hline Gritting & 16 & 15 & 1 & 0 & 0 & 100 \\
\hline Conjunctive hyperemia & 13 & 2 & 7 & 4 & 0 & 100 \\
\hline Palpebral edema & 18 & 2 & 7 & 7 & 2 & 89 \\
\hline
\end{tabular}

Regarding side effects, none of the 30 patients showed hyperpigmentation at the irradiated site.

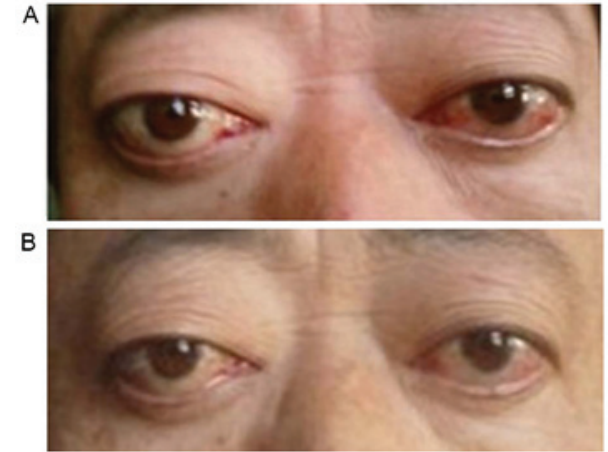

Figure 4. Graves' ophthalmopathy following external therapy with an in-house ${ }^{32} \mathrm{P}$ source in a 53-year-old male. (A) Prior to therapy; (B) 4 months post-therapy.

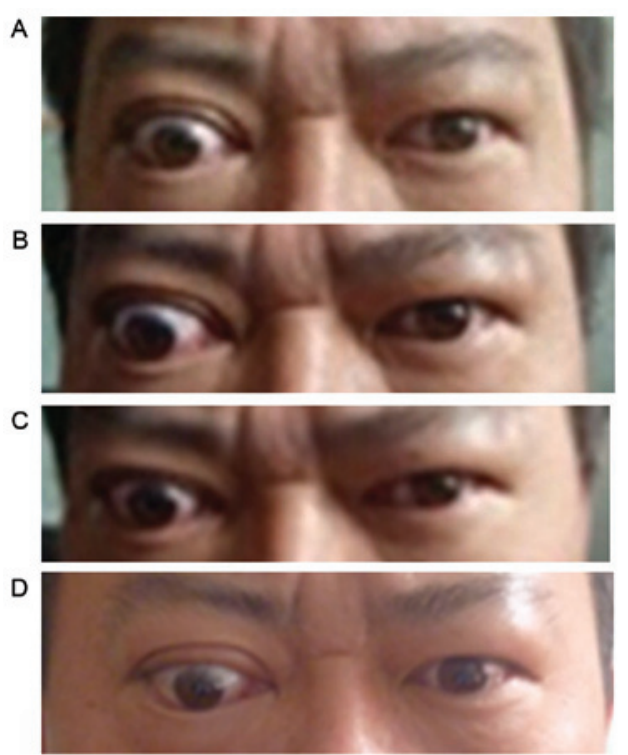

Figure 5. Graves' ophthalmopathy following external therapy with an in-house ${ }^{32} \mathrm{P}$ source in a 44-year-old male. (A) Prior to therapy; (B) $24 \mathrm{~h}$ after initial therapy; (C) 48 days after therapy; (D) 4 months after therapy.

There were no cataracts nor other serious complications during the follow-up period.
Table V. Comparison between changes in eye protrusion $(\mathrm{mm})$ by brachytherapy in the present study and orbital irradiation combined with retrobulbar injection of TAA reported by a previous study.

\begin{tabular}{lccc}
\hline Treatment & $\mathrm{N}$ & $\begin{array}{c}\text { Prior to } \\
\text { treatment }\end{array}$ & $\begin{array}{c}\text { After } \\
\text { treatment }\end{array}$ \\
\hline $\begin{array}{l}\text { Brachytherapy } \\
\text { Right eye }\end{array}$ & 30 & $18.17 \pm 4.60$ & $14.64 \pm 4.80$ \\
Left eye & 30 & $17.68 \pm 5.16$ & $14.32 \pm 4.87$ \\
$\begin{array}{l}\text { Orbital irradiation } \\
\text { + retrobulbar TAA }\end{array}$ & 46 & $17.90 \pm 2.10$ & $14.40 \pm 2.30$ \\
\hline
\end{tabular}

Values are expressed as the mean \pm standard deviation. TAA, triamcinolone actinide.

\section{Discussion}

Radiotherapy has an established place in the treatment of ophthalmic diseases such as conjunctivitis, pterygium, corneal neovascularization and conjunctiva capillary hemangioma. The present study examined the effect of ${ }^{32} \mathrm{P}$ brachytherapy in a cohort of 30 patients suffering from ophthalmopathy. The treatment was curative in all cases apart from two. In terms of swelling, most of the benefit showed by the fifth day of treatment and symptoms including pain, eye inflation, photophobia and lachrymation were also clearly reduced in the early stage of treatment, particularly pain and eye inflation. Based on the improvement in exophthalmos and associated symptoms, the effective rate of ${ }^{32} \mathrm{P}$ brachytherapy was $100 \%$. Only two patients relapsed during the follow-up period, which ranged from 3 to 12 months.

As the patient population consisted of individuals whose symptoms had persisted for at least 6 months after their thyroid function returned to normal, the improvements reported in the present study are likely to be due to the ${ }^{32} \mathrm{P}$ brachytherapy rather than a consequence of the thyroid treatment.

Brachytherapy with a sealed source is in principle the same as orbital irradiation from a linear accelerator, and each of the wo modalities has a curative effect on eye swelling, pain 
and light lacrimation, but not on ocular muscle involvement. A previous study reported that orbital irradiation resulted in a median exophthalmos reduction of $3 \mathrm{~mm}$ (11), whereas the present study reported average reductions of $3.36 \pm 1.73 \mathrm{~mm}$ (left eye) and 3.05 $\pm 2.04 \mathrm{~mm}$ (right eye). Regarding the effect on eye protrusion, there is no significant difference between the results of the present and those of a previously published study on orbital irradiation combined with retrobulbar injection of triamcinolone actinide (12). The comparison is summarized in Table V.

However, the present study did observe one difference in that the response to ${ }^{32} \mathrm{P}$ brachytherapy was a more rapid compared with that to orbital irradiation. For orbital irradiation, symptoms typically start to improve 1-2 weeks after treatment and exophthalmos reduction is rarely reported before one month after treatment $(11,13,14)$. The response to brachytherapy was more rapid, with eye inflation, photophobia and light lacrimation obviously improved on the day of treatment, and eyeball swelling clearly reduced by 10 days.

The difference between ${ }^{32} \mathrm{P}$ brachytherapy and orbital irradiation may be associated with the tissue being treated. There was no obvious improvement in eye pain and inflation when the retrobulbar space was irradiated. However, symptoms were obviously improved when the superior palpebra was irradiated. The most reasonable explanation of these rapid responses is that radiation inhibits nervous excitation, relieving eye inflation and pain and relaxing the extraocular muscles. In addition, radiation may retract the eyeball by relieving retrobulbar edema. The best evidence for this hypothesis is the rapid and effective relief of eyelid spasm. By contrast, retrobulbar radiotherapy does not directly irradiate the eyeball. It presumably only minimally inhibits sympathetic nervous excitation, since its effect is mainly through lymphokine secretion, leading to an increase in secreted glycosaminoglycan $(15,16)$. The time frame for such a mechanism is likely to be protracted.

The penetration depth of ${ }^{32} \mathrm{P} \beta$-particles in human tissue is only $8 \mathrm{~mm}$, which should preclude a therapeutic effect on most tissue. However, in an energy conversion process, $\beta$-particles can give rise to Bremsstrahlung $\mathrm{X}$-rays that would have a greater penetration depth.

Although the perspective of safety has priority during selection of the appropriate dose of ${ }^{32} \mathrm{P}$ brachytherapy, the optimal treatment dose must also be established. The original intention of adopting ${ }^{32} \mathrm{P}$ brachytherapy treatment was to relieve the continuous pain caused by gradually reducing the exophthalmos. The influence of the orbital irradiation dose has been investigated (17). Comparison of different dose and fractionation schemes (20 Gy/20 weeks; $10 \mathrm{~Gy} / 2$ weeks; $20 \mathrm{~Gy} / 2$ weeks) suggested that all such schemes were therapeutically equivalent. Moreover, Cardoso et al (11) gave $10 \mathrm{~Gy}$ over 10 weeks and also achieved a good curative effect. It is expected that ${ }^{32} \mathrm{P}$ brachytherapy requires a similar dose, while this requires further verification.

Side effects of ${ }^{32} \mathrm{P}$ brachytherapy are transient and without serious consequences. The main issue is that while ophthalmopathy symptoms first improve, they reappear after 3-5 days, although to a reduced degree, and these symptoms only persist for a further 2-4 days. For instance, pain aggravation occurred in only one case, appearing on the 4th day after the start of treatment and being relieved by the next day with no treatment break. No obvious reaction occurs in most cases if the dose delivered to the eye is within $20 \mathrm{~Gy}$. However, conjunctival injection, cellular edema and radiation damage appear with doses exceeding 20 Gy (10). As the irradiation dose was small, no hyperpigmentation was seen at the irradiated site.

Orbital irradiation for ophthalmopathy treatment is still in an early stage of development, and there are concerns about late side effects of orbital irradiation. Wakelkamp et al (5) conducted a long-term observation on the therapeutic response and normal tissue complications following retrobulbar irradiation, with an average observation period of $11 \pm 3$ years. Considering the potential morbidities of eye neoplasms, cataract, retinopathy and mortality between retrobulbar irradiation and hormone treatment groups, they showed that retrobulbar irradiation was safe and lacking any late toxicity. So far, there have been no reports of cancer after ${ }^{32} \mathrm{P}$ or ${ }^{90} \mathrm{Sr}$ treatment, leading to the conclusion that ${ }^{32} \mathrm{P}$ brachytherapy for ophthalmopathy is safe.

In conclusion, the present study demonstrated that ${ }^{32} \mathrm{P}$ brachytherapy is a simple procedure for Graves' ophthalmopathy arising from hyperthyroidism with a curative effect. While it is a promising therapeutic method, it was only applied it to a small group and issues of optimal dose and long term morbidity require further research.

\section{References}

1. Wyszomirska A: Iodine-131 for therapy of thyroid diseases. Physical and biological basis. Nucl Med Rev Cent East Eur 15: 120-123, 2012

2. Weiler DL: Thyroid eye disease: A review. Clin Exp Optom 100: 20-25, 2017.

3. Bartalena L, Baldeschi L, Boboridis K, Eckstein A, Kahaly GJ, Marcocci C, Perros P, Salvi M and Wiersinga WM; European Group on Graves' Orbitopathy (EUGOGO): The 2016 European thyroid association/European group on graves' orbitopathy guidelines for the management of graves' orbitopathy. Eur Thyroid J 5: 9-26, 2016.

4. Wu Y, Tong B, Luo Y, Xie G and Xiong W: Effect of radiotherapy on moderate and severe thyroid associated ophthalmopathy: A double blind and self-controlled study. Int J Clin Exp Med 8: 2086-2096, 2015.

5. Wakelkamp IM, Tan H, Saeed P, Schlingemann RO, Verbraak FD, Blank LE, Prummel MF and Wiersinga WM: Orbital irradiation for Graves' ophthalmopathy is it safe? A long-term follow-up study. Ophtalmology 111: 1557-1562, 2004.

6. Rajendram R, Bunce C, Lee RW and Morley AM: Orbital radiotherapy for adult thyroid eye disease. Cochrane Database Syst Rev 11: CD007114, 2012.

7. Prummel MF, Mourits MP, Blank L, Berghout A, Koornneef L and Wiersinga WM: Randomized double-blind trial of prednisone versus radiotherapy in Graves' ophthalmopathy. Lancet 342: 949-954, 1993.

8. Tsujino K, Hirota S, Hagiwara M, Fukada S, Takada Y, Hishikawa Y, Kono M and Abe M: Clinical outcomes of orbital irradiation combined with or without systemic high-dose or pulsed corticosteroids for Graves' ophthalmopathy. Int J Radiat Oncol Biol Phys 48: 857-864, 2000.

9. Marquez SD, Lum BL, McDougall IR, Katkuri S, Levin PS, MacManus M and Donaldson SS: Long-term results of irradiation for patients with progressive Graves' ophthalmopathy. Int J Radiat Oncol Biol Phys 51: 766-774, 2001.

10. Liang Z: Apply the paste treatment of nuclear medicine jinan. Jinan Press 10: 18-68, 2004.

11. Cardoso CC, Giordani AJ, Wolosker AM, Souhami L, Manso PG, Dias RS, Segreto HR and Segreto RA: Protracted hypofractionated radiotherapy for Graves' ophthalmopathy: A pilot study of clinical and radiologic response. Int J Radiat Oncol Biol Phys 82: 1285-1291, 2012.

12. Zheng $\mathrm{H}$ and Zhang L: Observation on orbital radiotherapy and retrobulbar injection for treatment of patients with Graves Ophthalmopathy. Yi Xue Lin Chuang Yan Jiu 7: 759-761, 2004. 
13. Marcocci C, Altea MA and Leo M: Treatment options for Graves orbitopathy. Expert Opin Pharmacother 13: 795-806, 2012.

14. Cai W and Qu S: Observation of curative effect of therapy of exophthalmos131i radiotherapy combined with retrobulbar. Shanghai Yi Xue 6: 379-380, 2001.

15. Li HX, Xiang N, Hu WK and Jiao XL: Relation between therapy options for Graves'disease and the course of Graves' ophthalmopathy: A systematic review and meta-analysis. J Endocrinol Invest 39: 1225-1233, 2016.
16. Karra E, Yousseif A, Rose GE and Baldeweg SE: Clinical assessment of patients with thyroid eye disease. Br J Hosp Med (Lond) 77: C2-C5, 2016.

17. Tanda ML and Bartalena L: Efficacy and safety of orbital radiotherapy for graves' orbitopathy. J Clin Endocrinol Metab 97: 3857-3865, 2012. 